OPEN ACCESS

Edited by: Baiquan Liu,

Sun Yat-Sen University, China

Reviewed by:

Gufeng $\mathrm{He}$,

Shanghai Jiao Tong University, China Huaibin Shen,

Henan University, China

${ }^{*}$ Correspondence: Wenqing Zhu

wqzhu@shu.edu.cn

Specialty section: This article was submitted to Nanoscience,

a section of the journal

Frontiers in Chemistry

Received: 23 January 2021 Accepted: 15 April 2021

Published: 29 April 2021

Citation:

Zhu W, Ding K, Yi C, Chen R, Wei B, Huang $L$ and Li J (2021) Use of Hybrid PEDOT:PSS/Metal Sulfide Quantum

Dots for a Hole Injection Layer in Highly Efficient Green Phosphorescent Organic Light-Emitting Diodes.

Front. Chem. 9:657557.

doi: 10.3389/fchem.2021.657557

\section{Use of Hybrid PEDOT:PSS/Metal Sulfide Quantum Dots for a Hole Injection Layer in Highly Efficient Green Phosphorescent Organic Light-Emitting Diodes}

\author{
Wenqing Zhu ${ }^{1,2 *}$, Kuangyu Ding ${ }^{1,2}$, Chen $\mathrm{Yi}^{1{ }^{1,2}}$, Ruilin Chen ${ }^{1,2}$, Bin Wei ${ }^{2}$, Lu Huang ${ }^{1}$ and \\ Jun $L i^{1}$ \\ ${ }^{1}$ School of Materials Science and Engineering, Shanghai University, Shanghai, China, ${ }^{2}$ School of Mechatronic Engineering and \\ Automation, Key Laboratory of Advanced Display and System Applications, Ministry of Education, Shanghai University, Shanghai, \\ China
}

In this study, we have synthesized the molybdenum sulfide quantum dots ( $\mathrm{MoS}_{2}$ QDs) and zinc sulfide quantum dots (ZnS QDs) and demonstrated a highly efficient green phosphorescent organic light-emitting diode (OLED) with hybrid poly (3,4ethylenedioxythiophene)/poly (styrenesulfonate) (PEDOT:PSS)/QDs hole injection layer (HIL). The electroluminescent properties of PEDOT:PSS and hybrid HIL based devices were explored. An optimized OLED based on the PEDOT:PSS/MoS 2 QDs HIL exhibited maximum current efficiency (CE) of $72.7 \mathrm{~cd} \mathrm{~A}^{-1}$, which shows a $28.2 \%$ enhancement as compared to counterpart with single PEDOT:PSS HIL. The higher device performance of OLED with hybrid HIL can be attributed to the enhanced hole injection capacity and balanced charge carrier transportation in the OLED devices. The above analysis illustrates an alternative way to fabricate the high efficiency OLEDs with sulfide quantum dots as a HIL.

Keywords: phosphorescent organic light-emitting diodes, metal sulfide QDs, hybrid hole injection layer, PEDOT: PSS, ZnS, $\mathrm{MoS}_{2}$

\section{INTRODUCTION}

New-generation organic light-emitting diodes (OLEDs) have attracted huge research interests due to their unique properties such as high color purity, light weight and flexibility (Liao et al., 2004; Wen et al., 2005; Wang et al., 2018; Wang et al., 2020). One of the most important issue for the OLEDs application in industry is the device efficiency. The effective charge injection and transportation, and exciton confinement in the emitting layer are the key parameters to achieve highly efficient OLEDs (Lee et al., 1999; Wang et al., 2008; Song et al., 2018; Zhao et al., 2020). To date, many research groups have used the solution processed hole injection layer (HIL) to improve the device performance and to decrease the fabrication costs (Li et al., 2018; Hu et al., 2020; Wang et al., 2020). To obtain the highly efficient OLEDs, the overall requirements on solution processed HIL should possess excellent optical and electrical characteristics such as high transparency and conductivity, as well as the low surface roughness (Zhao et al., 2016; McEwan et al., 2018; Feng et al., 2020). Therefore, the synthesis and development of solution processed hole injection materials are very important to achieve highperformance devices. 


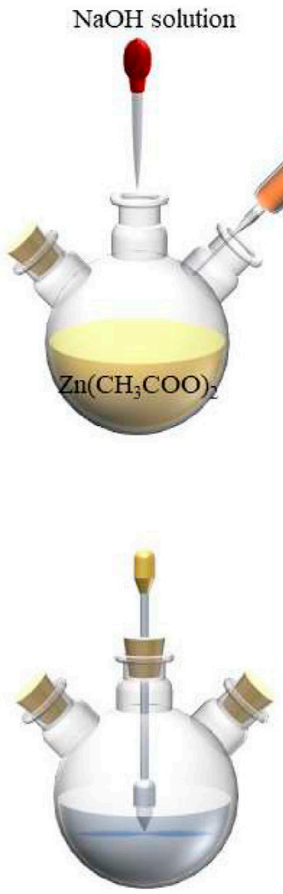

Rotation at $80^{\circ} \mathrm{C} / 1.5 \mathrm{~h}$

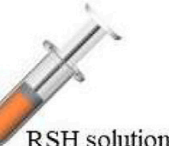

$\mathrm{SH}$ solution
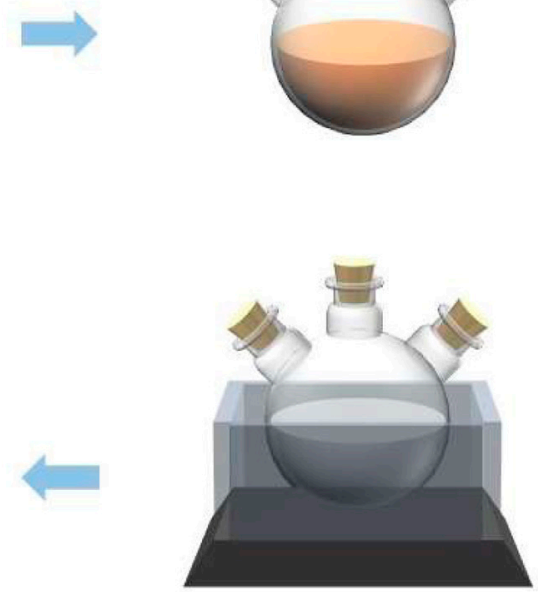

Ultrasonic $30 \mathrm{~min}$

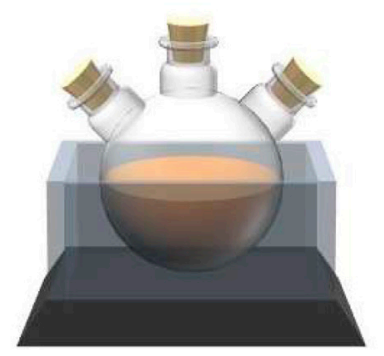

Ultrasonic $10 \mathrm{~min}$

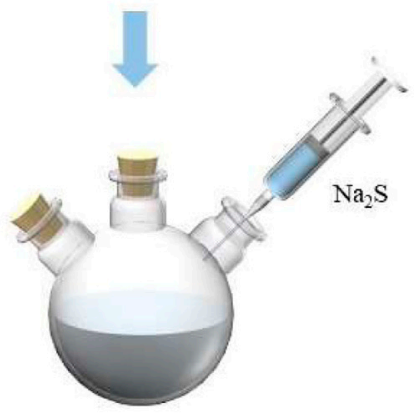

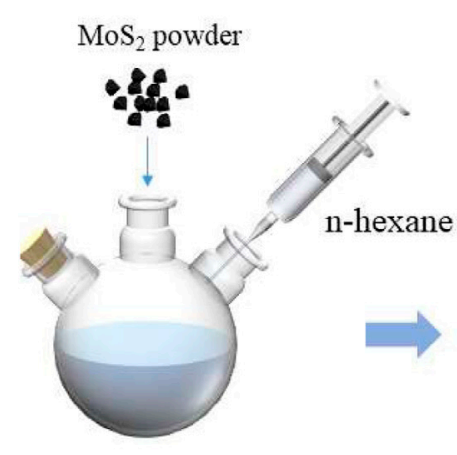
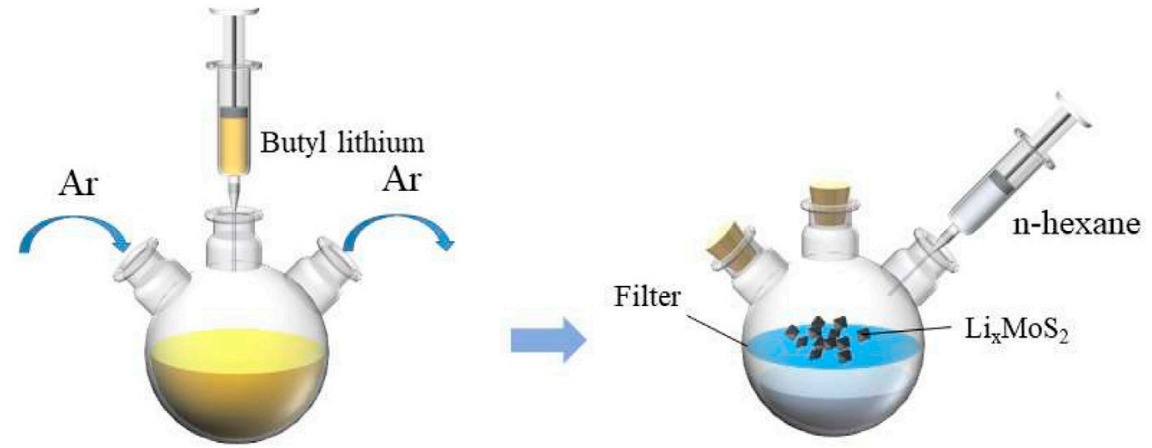

Sealed with $48 \mathrm{~h}$
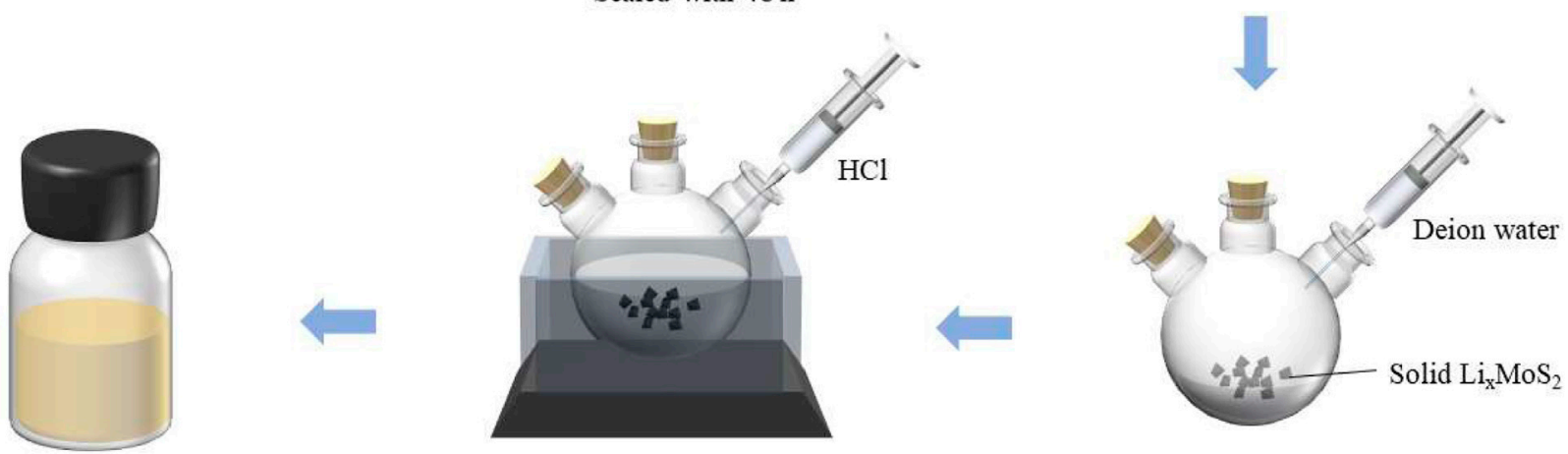

Ultrasonic $1 \mathrm{~h}$

$\mathrm{MoS}_{2} \mathrm{QDs}$

FIGURE 1 | Schematic of ZnS QDs (upper) and $\mathrm{MoS}_{2}$ QD (below) synthesis procedure.

The traditional poly (3,4-ethylenedioxythiophene)/poly (styrenesulfonate) (PEDOT:PSS) is widely used as a solution processed HIL in organic electronics (Benor et al., 2010;
Salsberg and Aziz, 2019). However, its acidic property and easily absorption for water in the air significantly deteriorate the device performance. The traditional molybdenum oxide 
$\left(\mathrm{MoO}_{\mathrm{x}}\right)$ is also employed in the OLED devices as an effective HIL due to its matched work function with ITO electrode and low surface roughness and high transparency. However, the $\mathrm{MoO}_{\mathrm{x}}$ as HIL is normally prepared by using vacuum thermal evaporation method which is not a good candidate for low-cost OLED preparation. More importantly, to date, there is seldom reports related to the solution-processed $\mathrm{MoO}_{\mathrm{x}}$ in electronic devices because of the limited solubility. Recently, solution processed sulfide quantum dots (QDs) such as zinc sulfide QDs (Zn QDs), molybdenum sulfide QDs ( $\left.\mathrm{MoS}_{2} \mathrm{QDs}\right)$, and others as HILs/HTLs have been reported as an effective way to increase charge injection due to their unique properties, such as high solubility, tunable work function and low cost, resulting in the enhanced devices performance (Kim et al., 2015; Lenkeviciute et al., 2015; Xie et al., 2019). In our previous work, we have reported a highly efficient inverted OLEDs by using the solution processed ZnS QDs as an electron injection layer due to the excellent optical and electrical properties (Shi et al., 2019).

In this paper, the application of PEDOT:PSS doped with various QDs as the hybrid HIL for high-efficiency green phosphorescent OLED devices have been studied. And the hybrid PEDOT:PSS/QDs HILs were investigated based on their optical and morphological characteristics, as well as the surface energy. We found that the hybrid HIL based device revealed relatively higher device performance with the best current efficiency (CE) of $72.7 \mathrm{~cd} \mathrm{~A}^{-1}$, which was a $28.2 \%$ enhancement as compared to the neat PEDOT:PSS based devices.

\section{EXPERIMENTAL}

\section{General Information}

The organic functional molecules were obtained from e-Ray Optoelectronics Corp (China). The hole injection material poly (3,4-ethylenedioxythiophene)/poly (styrenesulfonate) (PEDOT: PSS) was purchased from Heraeus, Germany. Indium tin oxide (ITO, $15 \mathrm{Ohm}$ per sheet, $150 \mathrm{~nm}$ )-coated glass substrates were ordered from CSG Holding Co. Ltd. (China). All chemicals and reagents in this work were used as received from commercial sources without further purification unless otherwise stated.

\section{Synthesis of Zinc Sulfide Quantum Dots and Molybdenum Sulfide Quantum Dots}

ZnS QDs were synthesized using our previously reported method (Shi et al., 2019). As shown in Figure 1, in brief, take out $2.4018 \mathrm{~g}$ $\mathrm{Na}_{2} \mathrm{~S} \cdot 9 \mathrm{H}_{2} \mathrm{O}$ and put it into a $100 \mathrm{ml}$ volumetric flask. add distilled water to $100 \mathrm{ml}$ scale, mix well and keep well. Then $0.02 \mathrm{~mol} \mathrm{~L}^{-1}$ RSH aqueous solution and $0.1 \mathrm{~mol} \mathrm{~L}^{-1} \mathrm{Zn}\left(\mathrm{CH}_{3} \mathrm{COO}\right)_{2}$ solution was prepared. The above $\mathrm{Na}_{2} \mathrm{~S}$ aqueous solution is the same. Add $25 \mathrm{ml}$ of $0.1 \mathrm{~mol}^{-1} \mathrm{Zn}\left(\mathrm{CH}_{3} \mathrm{COO}\right)_{2}$ solution above into a three necked round bottom flask, continue to add $50 \mathrm{ml}$ of $0.02 \mathrm{~mol}^{-1}$ ethanoic acid, and then dissolve it with $0.5 \mathrm{~mol} \mathrm{~L}^{-1} \mathrm{NaOH}$. Finally, after ultrasonic treatment for $10 \mathrm{~min}$, the prepared $\mathrm{Na}_{2} \mathrm{~S}$ aqueous solution was added rapidly to $5 \mathrm{ml}$, and then the device was ultrasonic treated for $30 \mathrm{~min}$. Finally, $\mathrm{ZnS}$ nano quantum dots were prepared by stirring at $80^{\circ} \mathrm{C}$ for $1 \mathrm{~h}$ and $30 \mathrm{~min}$.
For the synthesis of $\mathrm{MoS}_{2}$ QDs, firstly, taking $1 \mathrm{~g}$ of $\mathrm{MoS}_{2}$ powder into a three-necked round bottom flask, add $12 \mathrm{ml}$ of $\mathrm{n}$-hexane, and passed the protective gas argon into the flask. The three-necked round bottom flask was immediately sealed and allowed to stand for $48 \mathrm{~h}$. The LixMoS 2 in the intercalation was vacuum filtered. The product obtained by suction filtration was repeatedly washed with n-hexane solution to remove excess butyl lithium and organic residues. Then quickly took out $\mathrm{LixMoS}_{2}$ on the suction filter membrane and made it react with deionized water. The solution formed by the reaction of $\mathrm{LixMoS}_{2}$ with water was placed in an ultrasonic microwave instrument for auxiliary ultrasound for $1 \mathrm{~h}$. The $\mathrm{MoS}_{2}$ flakes in the suspension would quickly produce black flocculent precipitates, and then centrifuged with deionized water for several times to adjust the $\mathrm{pH}$ of the solution to medium which could remove $\mathrm{Li}, \mathrm{Cl}$ ions and organic residues and obtain the $\mathrm{MoS}_{2} \mathrm{QD}$ solution.

\section{Device Fabrication}

Devices were fabricated with a configuration of ITO/PEDOT: PSS $(40 \mathrm{~nm}) / \mathrm{NPB} \quad(30 \mathrm{~nm}) / \mathrm{TCTA} \quad(10 \mathrm{~nm}) / \mathrm{mCP}: \quad 5 \% \mathrm{Ir} \quad(\mathrm{ppy})_{3}$ $(20 \mathrm{~nm}) / \mathrm{TPBi}(35 \mathrm{~nm}) / \mathrm{Liq}(1 \mathrm{~nm}) / \mathrm{Al}(100 \mathrm{~nm})$, as shown in Figure 2, where ITO is the anode; PEDOT: PSS is the hole injection layer; $\quad \mathrm{N}, \mathrm{N}^{\prime}$-bis(naphthalen-1-yl)-N, $\mathrm{N}^{\prime}$-bis(phenyl)benzidine (NPB) and 4,4',4'-tris(carbazol-9-yl)triphenylamine (TCTA) are the hole transporting layer. 1,3-bis(N-carbazolyl) benzene $(\mathrm{mCP})$ is the host for green phosphorescent dopant; tris(2-phenyl-3-methyl-pyridine)iridium ( $\left.\operatorname{Ir}(\mathrm{ppy})_{3}\right)$ is the green dopant; 1,3,5-tris(1-phenyl-1H-benzimidazol-2-yl)benzene (TPBi) functions as the electron transporting layer and interlayer; Liq and $\mathrm{Al}$ are the electron injection layer and the cathode, respectively. The patterned ITO glass substrates were first cleaned sequentially by using detergent, deionized water, acetone, isopropanol and treated with a UV-ozone environment for about $20 \mathrm{~min}$. Then a $40 \mathrm{~nm}$ QDs doped PEDOT:PSS was spin-coated onto the ITO surface under the conditions of rotation speed of 3,000 rpm and spin coating time of $60 \mathrm{~s}$. After the spin coating process was completed, the ITO covering part of the electrode was wiped off with deionized water and being baked at $130^{\circ} \mathrm{C}$ for $20 \mathrm{~min}$ under air conditions. Then the substrates were transferred into a vacuum chamber. Then, organic layers and a metal cathode layer were successively deposited by using shadow masks to finish the device fabrication in a vacuum chamber under a base pressure less than $4 \times 10^{-6}$ mbar. The deposition rates for the organic layers and Al cathode were typically $2.0 \AA \mathrm{s}^{-1}$ and $5.0 \AA \mathrm{s}^{-1}$, respectively. The active area of OLEDs is $2 \times 2 \mathrm{~mm}^{2}$.

\section{Film and Device Characterization}

The transmittance spectra were recorded on a UV-2501PC spectrophotometer at room temperature. Drop shape analysis (Kino optical CA and interface tensiometer) was used to measure the contact angles of deionized (DI) water. The surface morphological images of the various HILs were analyzed in air by using AFM (Bruker, Santa Barbara, CA, USA) in a tapping mode. The EL characteristics were measured using a Keithley 2,400 source meter and a PR650 Spectra Colorimeter. The luminance and spectra of each device were measured in the direction perpendicular to the substrate. All the device characterization steps were carried out at room temperature under ambient laboratory conditions without encapsulation. 

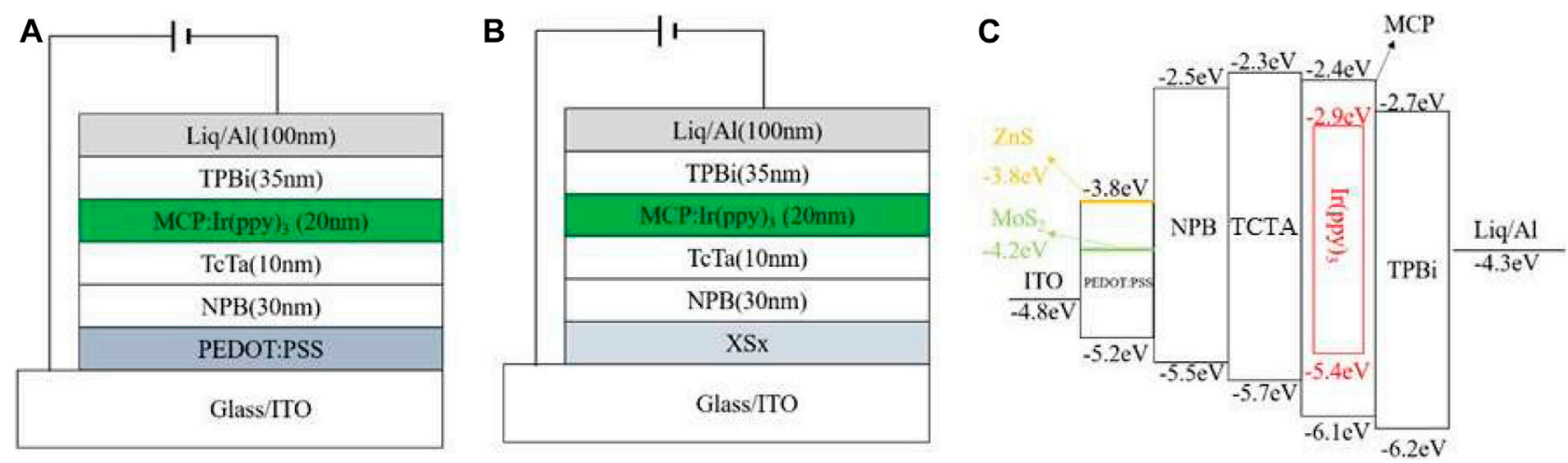

FIGURE 2 | Structure of the green phosphorescent OLEDs with (A) neat PEDOT:PSS HIL and (B) hybrid HIL. (C) The corresponding energy level diagram of the devices.
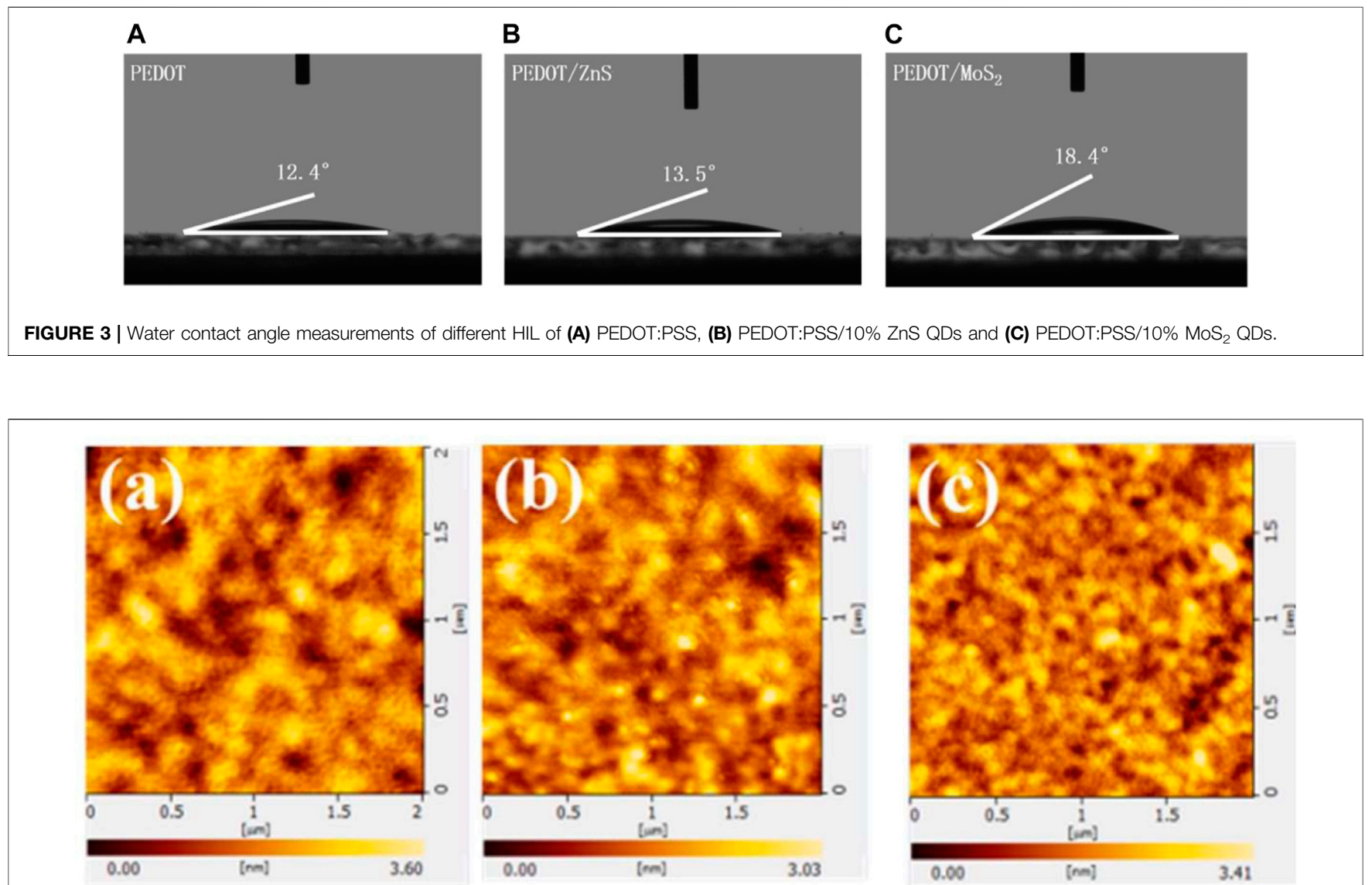

FIGURE 4 | AFM test of HILs of (A) PEDOT:PSS, (B) PEDOT:PSS/10\%ZnS QDs and (C) PEDOT:PSS/10\% MoS 2 QDs.

\section{RESULTS AND DISCUSSION}

Firstly, the water contact angles of neat PEDOT:PSS and hybrid PEDOT:PSS/QDs with the concentration of $10 \%(\mathrm{v} / \mathrm{v})$ on the glass substrate were measured to identify the hydrophilic and spread-ability of the resulting HILs, which could affect the film- forming property of hole transporting layer (HTL) (Yu et al., 2014; Singh et al., 2014; Huang et al., 2009; Cho et al., 2014). Figure 3 demonstrate the contact angles of water with PEDOT: PSS and PEDOT:PSS/QDs with different concentration are $12.4^{\circ}$, $13.5^{\circ}$ and $18.4^{\circ}$, respectively. The small contact angles reveal that the hybrid PEDOT:PSS/QDs HIL has strong hydrophilic and 


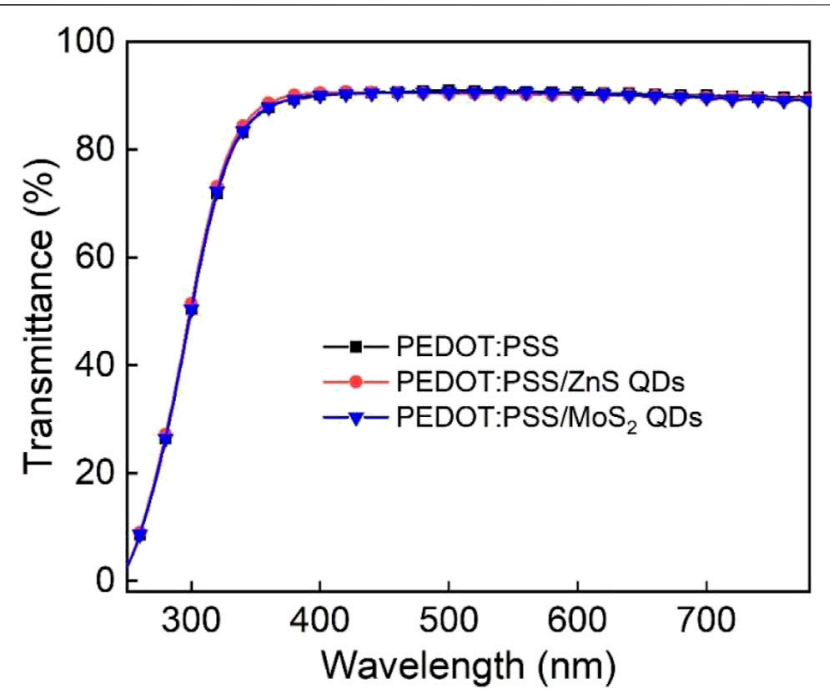

FIGURE 5 | The optical transmittance spectra for neat PEDOT:PSS PEDOT:PSS/10\% ZnS QDs and PEDOT:PSS/10\% MoS $_{2}$ QDs films.

spread-ability, resulting in a better interface and adhesion between anode and hole transporting layer (Phatak et al., 2012; Tsai et al., 2020).

The OLEDs are multi thin-film structures with the overall device thickness of around $200 \mathrm{~nm}$. Therefore, it is an essential requirement for OLED devices with a smooth surface on the substrate to avoid the trap states such as defects, shorts, and pinholes (Lee et al., 2018; Michels et al., 2021; Lim et al., 2008). Figure 4 shows the surface morphology profiles of glass/HIL (40 nm) observed by AFM measurements. The values of surface roughness (RMS) of neat PEDOT:PSS, PEDOT:PSS/10\%ZnS QDs and PEDOT:PSS/10\% $\mathrm{MoS}_{2}$ QDs film are 0.57, 0.47, and $0.69 \mathrm{~nm}$, respectively. The hybrid HIL films exhibit a low surface roughness, which result in more efficient hole injection and consequently affect the HTL and device performance (Kim et al., 2019; Ma et al., 2019).

Figure 5 shows the ultraviolet visible (UV-Vis) spectra measurement with the wavelength range of $220-800 \mathrm{~nm}$. The transmittances of the resulting film are over $90 \%$ in the visible region. Within the green emission spectral range, the glass substrate with hybrid PEDOT:PSS/ZnS QDs and PEDOT:PSS/ $\mathrm{MoS}_{2}$ QDs film with the concentration of $10 \%$ yielded a higher transmittance of $93 \%$ at $525 \mathrm{~nm}$ which is beneficial for enhancing the light extraction in OLED devices with normal configuration (Yang et al., 2014; Chiu and Chuang, 2015). The above results demonstrate the good visible light transmittance property of sulfide QDs-based thin films.

To evaluate the solution-processed hybrid PEDOT:PSS/ZnS QDs and PEDOT:PSS/MoS 2 QDs films as the HIL, the OLED devices with normal configuration were fabricated using the following structure: ITO/HIL $(40 \mathrm{~nm}) / \mathrm{NPB} \quad(30 \mathrm{~nm}) / \mathrm{TCTA}$ $(10 \mathrm{~nm}) / \mathrm{mCP}: 5 \% \operatorname{Ir}(\mathrm{ppy})_{3}(20 \mathrm{~nm}) / \mathrm{TPBi}(35 \mathrm{~nm}) / \mathrm{Liq}(1 \mathrm{~nm}) /$ $\mathrm{Al}(100 \mathrm{~nm})$. Here, the device with neat PEDOT:PSS as HIL was also fabricated as the reference device. The corresponding energy level diagram of OLED devices are also depicted in Figure 2. The current density $(J)$-voltage $(V)$-brightness $(L)$ properties of the OLEDs are exhibited in Figure $\mathbf{6}$ and the main electroluminescent properties are summarized in Table $\mathbf{1 .}$

The utilization of PEDOT:PSS/ZnS QDs based HILs significantly improved the device performances. From the $J-V$ characteristics of the devices with various concentrations of QDs based HILs, the current density increased compared with the neat PEDOT:PSS based device. In addition, the device with hybrid PEDOT:PSS/ZnS QDs HILs showed a higher luminance at same current density, as shown in Figure 6A. It is also noteworthy that the maximum current efficiency of the device with hybrid PEDOT:PSS/ZnS QDs is higher than that of the device with neat PEDOT:PSS. The PEDOT:PSS/5\%ZnS QDs based device showed excellent current efficiency (CE) of $63.8 \mathrm{~cd} \mathrm{~A}^{-1}$, which is superior to that of device with neat PEDOT:PSS of $55.1 \mathrm{~cd} \mathrm{~A}^{-1}$. It suggests that the hybrid PEDOT:PSS/ZnS QDs as HIL can significantly enhance hole injection capacity in comparison with the neat PEDOT:PSS HIL. The hybrid PEDOT:PSS $/ 10 \%$ ZnS QDs based OLED reaches a maximum luminance of $49,005 \mathrm{~cd} \mathrm{~m}^{-2}$ at $9.5 \mathrm{~V}$, among the other devices. Typical hybrid PEDOT:PSS/ZnS QDs based OLED gives emission with an EL peak of $516 \mathrm{~nm}$, as shown in Figure 6D.

Finally, we investigated the device performances based on the hybrid PEDOT:PSS/MoS 2 QDs as the HIL. The results are shown in Figure 7, and the performance parameters are also listed in Table 1. It should be noted that device with hybrid PEDOT:PSS/ $10 \% \mathrm{MoS}_{2}$ QDs shows the better performance compared with other devices. The device with the concentration of $10 \% \mathrm{MoS}_{2}$ QDs in PEDOT:PSS exhibited maximum CE of $72.7 \mathrm{~cd} \mathrm{~A}^{-1}$ and maximum luminescence of $46,354 \mathrm{~cd} \mathrm{~m}^{-2}$ with a low turn-on voltage of $3.6 \mathrm{~V}$. We also demonstrate that PEDOT:PSS doped with $10 \% \mathrm{MoS}_{2}$ QDs forms more higher quality film with lower surface roughness, which are beneficial to form a better interface and adhesion between anode and hole transporting layer. (Phatak et al., 2012; Tsai et al., 2020).

To further investigate the mechanisms of the different HILs in the OLED devices, we prepared hole-only devices (HODs) with device structures of ITO/HIL $(40 \mathrm{~nm}) / \mathrm{NPB} \quad(100 \mathrm{~nm}) / \mathrm{Al}$ $(100 \mathrm{~nm})$ with various hole injection layers (traditional PEDOT:PSS, PEDOT:PSS/10\%ZnS QD, and PEDOT:PSS/10\% $\left.\mathrm{MoS}_{2} \mathrm{QD}\right)$ and the current density-voltage $(J-V)$ characteristics of HODs based on the mixed HILs with the concentration of $10 \%$ were compared. The related results are shown in Figure 8. The HOD with the mixed PEDOT:PSS/QD HILs showed much higher current density than the devices with the traditional PEDOT:PSS HIL at the same driving voltages, indicating the more efficient hole injection.

The hole mobility based HOD was calculated from space charge limited current method by using the following MottGurney Law equation (Tang et al., 2019):

$$
J=\frac{9}{8} \varepsilon \varepsilon_{0} \mu \frac{V^{2}}{L^{3}} \exp \left(\beta \sqrt{\frac{V}{L}}\right),
$$

where $\varepsilon_{0}$ is the vacuum permittivity, $\varepsilon$ is the relative dielectric constant, $\beta$ is the Poole-Frankel factor, and $L$ is the thickness of HIL. The mobility of NPB $\left(1.6 \times 10^{-5} \mathrm{~cm}^{2} / \mathrm{V} \cdot \mathrm{S}^{-1}\right)$ is much lower 

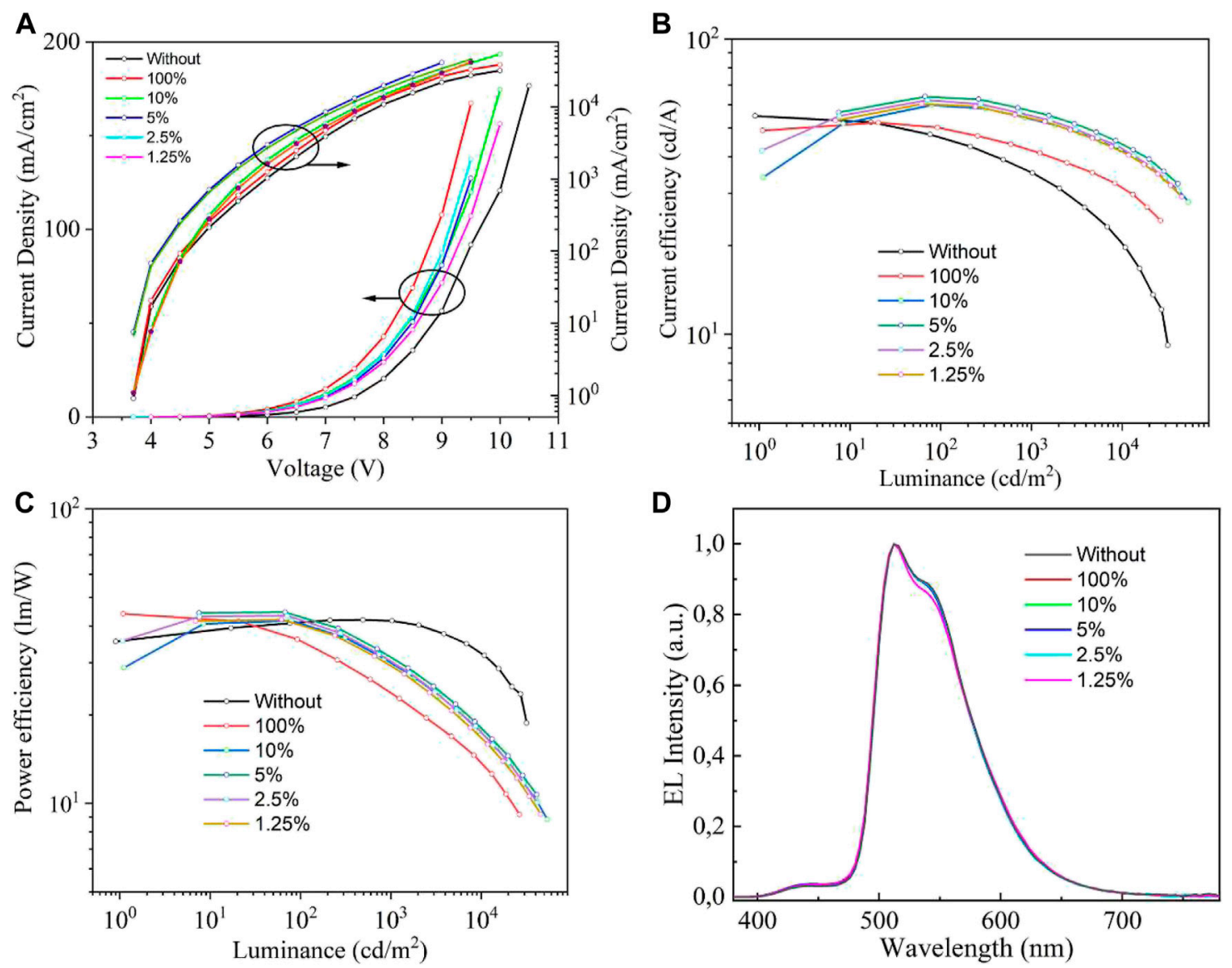

FIGURE 6 | The green phosphorescent OLED device with hybrid PEDOT:PSS/ZnS QDs film as the HIL. (A) Current density-voltage-brightness (J-V-L), (B) Current efficiency-luminance (CE-L), (C) Power efficiency-current density (PE-J) and (d) EL spectra at $6 \mathrm{~V}$ of the resulting devices with different concentrations.

TABLE 1 | Performance parameters of the green phosphorescent OLED device with the various HILs.

\begin{tabular}{|c|c|c|c|c|}
\hline HIL & $v_{\text {on }}[\mathrm{V}]^{\mathrm{a}}$ & $n_{\max }\left(\mathrm{cd} \mathrm{A}^{-1}\right)^{\mathrm{b}}$ & $L_{\max }\left(\mathrm{cd} \mathrm{m^{-2 }}\right)^{\mathrm{c}}$ & $n_{\max }\left(\operatorname{Im~W^{-1}}\right)^{\mathrm{d}}$ \\
\hline Neat PEDOT:PSS & 4 & 55.1 & 35,962 & 41.9 \\
\hline PEDOT:PSS/100\% ZnS & 4.2 & 52.2 & 46,354 & 43.9 \\
\hline PEDOT:PSS/10\% ZnS & 4.3 & 59.7 & 49,005 & 41.6 \\
\hline PEDOT:PSS/2.5\% ZnS & 4.3 & 62.1 & 41,844 & 43.3 \\
\hline PEDOT:PSS/1.25\% ZnS & 4.3 & 60.2 & 45,705 & 42.0 \\
\hline PEDOT:PSS/100\% $\mathrm{MoS}_{2}$ & 3.6 & 55.8 & 37,950 & 40.7 \\
\hline PEDOT:PSS/5\% $\mathrm{MoS}_{2}$ & 3.6 & 65.3 & 39,424 & 45.6 \\
\hline PEDOT:PSS/2.5\% $\mathrm{MoS}_{2}$ & 3.8 & 61.1 & 45,320 & 38.3 \\
\hline PEDOT:PSS/1.25\% $\mathrm{MoS}_{2}$ & 3.8 & 56.7 & 43,802 & 39.6 \\
\hline
\end{tabular}

${ }^{a}$ The operating voltage at a brightness of $1 \mathrm{~cd} \mathrm{~m}^{-2}$.

${ }^{b}$ The maximum $C E$.

${ }^{c}$ The maximum luminance.

${ }^{d}$ The maximum $P E$.

than that of PEDOT:PSS/QD. (Blom et al., 2005; Zhao et al., 2019) Thus, in the initial SCLC region, the hole mobility of PEDOT: PSS/QD based HODs is limited by the mobility of NPB. The hole mobility of PEDOT:PSS/QD based HOD was evaluated from the linear fitting the SCLC region (based on $\varepsilon=11.9, \varepsilon_{0}=8.85 \times 10^{-12}$ $\mathrm{Fm}^{-1}$, and $\left.\mathrm{L}=40 \mathrm{~nm}\right)$, which results in $\mu_{1}=1.748 \times 10^{-4} \mathrm{~cm}^{2} /$ 

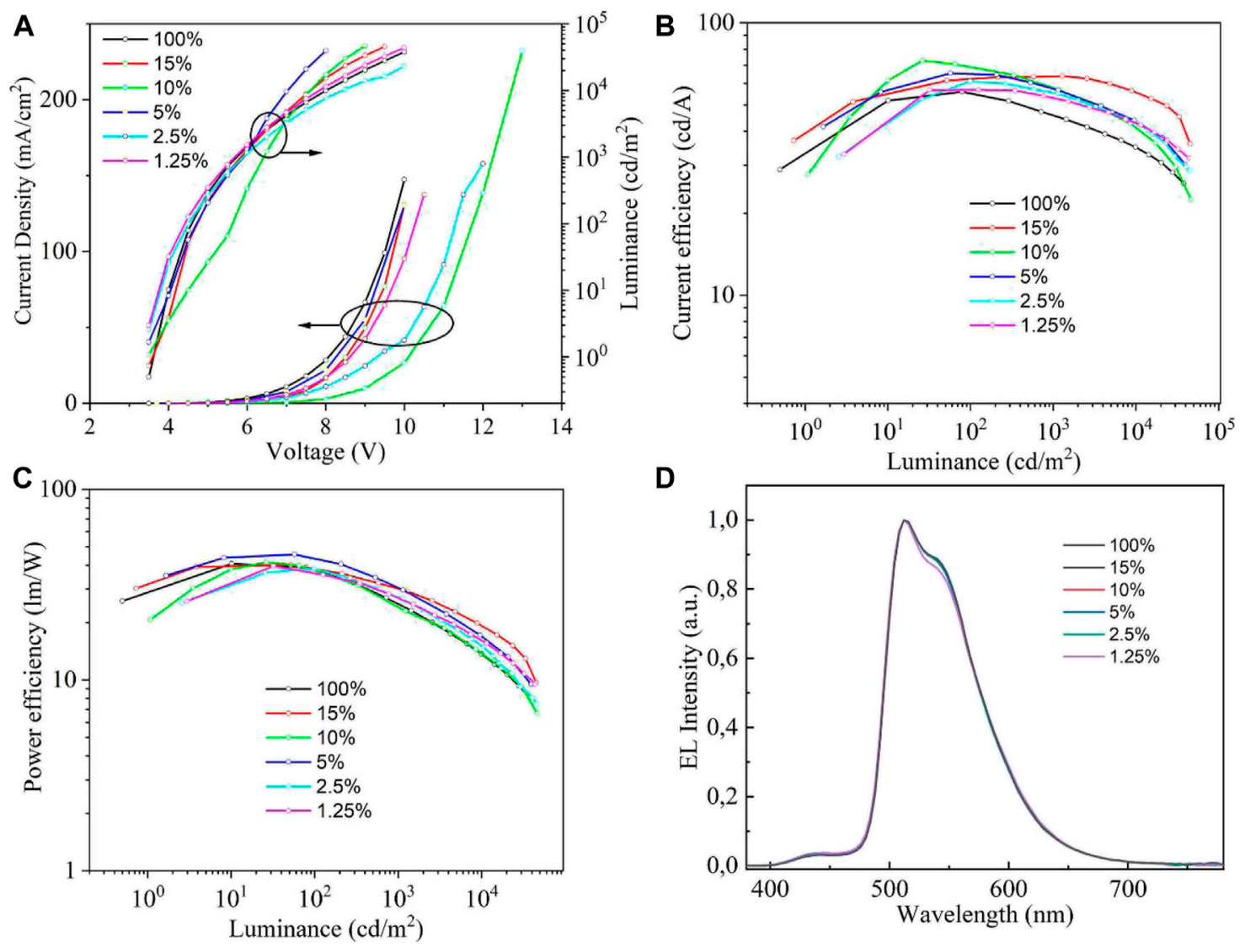

FIGURE 7|Device performance with hybrid PEDOT:PSS/MoS ${ }_{2}$ QDs film as the HIL. (A) J-V-L, (B) CE- $L$, (C) PE-J and (D) EL spectra at 6 V of the resulting devices with different concentrations.

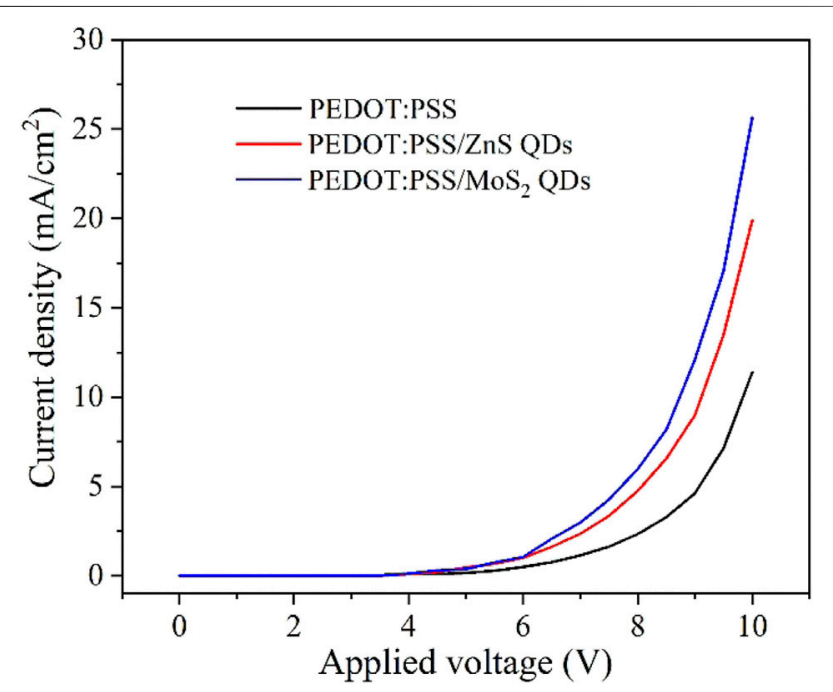

FIGURE 8 | The $\mathrm{J}$ - $\mathrm{V}$ characteristics of hole-only devices of ITO/HIL $(40 \mathrm{~nm}) /$ NPB $(100 \mathrm{~nm}) / \mathrm{Al}(100 \mathrm{~nm})$.

$\mathrm{V} \cdot \mathrm{S}^{-1}$ (PEDOT:PSS), $\mu_{2}=7.67 \times 10^{-4} \mathrm{~cm}^{2} / \mathrm{V} \cdot \mathrm{S}^{-1}$ (PEDOT:PSS/ $\mathrm{ZnS}$ QD), $\mu_{3}=1.464 \times 10^{-3} \mathrm{~cm}^{2} / \mathrm{V} \cdot \mathrm{S}^{-1}$ (PEDOT:PSS $\left./ \mathrm{MoS}_{2} \mathrm{QD}\right)$, indicating that the mixing of QD and PEDOT:PSS can improve the hole mobility compared to PEDOT:PSS based OLED obviously. This result is coincidence with device performance, which is responsible for the improvement of hole injection in OLEDs. Therefore, based on the corresponding energy level diagram of devices and the analytic results of SCLC method of HODs, the enhanced device efficiency with hybrid HILs can be attributed to the reduced hole injection barrier and charge recombination, resulting in the overall performance improvement of the resulting OLEDs. (Li and Marks, 2008; Han et al., 2015; Yadav et al., 2020)

\section{CONCLUSION}

In this work, we have systematically investigated the optical and electrical properties of the $\mathrm{ZnS}$ and $\mathrm{MoS}_{2}$ QDs. We have demonstrated that hybrid PEDOT:PSS/QDs film as the HIL in conventional OLED could highly enhance hole injection from anode into organic hole transporting layer, and consequently improves the device efficiency. Furthermore, the device with hybrid HILs shows lower turn-on voltage and higher luminance because of its enhanced hole injection property. Among all the devices with various HILs, the device with hybrid PEDOT:PSS/MoS 2 QDs HIL showed a lowest turn-on voltage of $3.6 \mathrm{~V}$ and the highest maximum $\mathrm{CE}$ of $72.7 \mathrm{~cd} \mathrm{~A}{ }^{-1}$, 
achieving an enhancement of $28.2 \%$ than those with neat PEDOT:PSS based devices. We conclude that the improved film morphology of the hybrid HILs, balanced charge carrier injection and recombination are important factors to contribute the high performance of OLEDs.

\section{DATA AVAILABILITY STATEMENT}

The raw data supporting the conclusions of this article will be made available by the authors, without undue reservation.

\section{REFERENCES}

Benor, A., Takizawa, S.-y., Pérez-Bolívar, C., and Anzenbacher, P., Jr (2010). Efficiency Improvement of Fluorescent OLEDs by Tuning the Working Function of PEDOT:PSS Using UV-Ozone Exposure. Org. Electronics 11 (5), 938-945. doi:10.1016/j.orgel.2010.02.014

Blom, P. W. M., Tanase, C., De Leeuw, D. M., and Coehoorn, R. (2005). Thickness Scaling of the Space-Charge-Limited Current in Poly (P-phenylene Vinylene). Appl. Phys. Lett. 86 (9), 092105. doi:10.1063/1.1868865

Chiu, T. L., and Chuang, Y. T. (2015). Spectral Observations of Hole Injection with Transition Metal Oxides for an Efficient Organic Light-Emitting Diode. J. Phys. D: Appl. Phys. 48 (7), 075101. doi:10.1088/0022-3727/48/7/075101

Cho, A. R., Kim, S. H., Lee, E.-W., Gwak, G., Jang, J., and Park, L. S. (2014). Flexible OLED Fabricated on Glass Fabric Reinforced Film and Performance. Mol. Crystals Liquid Crystals 602 (1), 26-33. doi:10.1080/15421406.2014.944365

Feng, C., Zheng, X., Xu, R., Zhou, Y., Hu, H., Guo, T., and Li, F. (2020). Highly Efficient Inkjet Printed Flexible Organic Light-Emitting Diodes with Hybrid Hole Injection Layer. Org. Electronics 85, 105822. doi:10.1016/j.orgel.2020. 105822

Han, T.-H., Song, W., and Lee, T.-W. (2015). Elucidating the Crucial Role of Hole Injection Layer in Degradation of Organic Light-Emitting Diodes. ACS Appl. Mater. Inter. 7 (5), 3117-3125. doi:10.1021/am5072628

Hu, Y., Song, L., Zhang, S., Lv, Y., Lin, J., Guo, X., et al. (2020). Improving the Efficiency of Multilayer Organic Light-Emitting Transistors by Exploring the Hole Blocking Effect. Adv. Mater. Inter. 7 (17), 2000657. doi:10.1002/admi. 202000657

Huang, Z. H., Zeng, X. T., Sun, X. Y., Kang, E. T., Fuh, J. Y. H., and Lu, L. (2009). Influence of Electrochemical Treatment of ITO Surface on Nucleation and Growth of OLED Hole Transport Layer. Thin solid films 517 (17), 4810-4813. doi:10.1016/j.tsf.2009.03.020

Kim, G.-E., Shin, D.-K., Lee, J.-Y., and Park, J. (2019). Effect of Surface Morphology of Slot-Die Heads on Roll-To-Roll Coatings of Fine PEDOT:PSS Stripes. Org. Electronics 66, 116-125. doi:10.1016/j.orgel.2018.12.033

Kim, H. J., Shin, M. H., Hong, H. G., Song, B. S., Kim, S. K., Koo, W. H., and Kim, Y. J. (2015). Enhancement of Optical Efficiency in White OLED Display Using the Patterned Photoresist Film Dispersed with Quantum Dot Nanocrystals. J. Display Technology 12 (6), 526-531.

Lee, J., Kim, G., Shin, D.-K., Seo, Y., Kim, K., and Park, J. (2018). Improved Surface Morphology of Crosslinked Hole Transport Films by a Mixture of Polymer for OLEDs. IEEE Trans. Electron. Devices 65 (8), 3311-3317. doi:10.1109/ted.2018. 2842130

Lee, S. T., Wang, Y. M., Hou, X. Y., and Tang, C. W. (1999). Interfacial Electronic Structures in an Organic Light-Emitting Diode. Appl. Phys. Lett. 74 (5), 670-672. doi:10.1063/1.122982

Lenkeviciute, B., Vitkus, M., Juska, G., and Genevicius, K. (2015). Hybrid OLEDs with CdSSe1-/ZnS Core-Shell Quantum Dots: An Investigation of Electroluminescence Properties. Synth. Met. 209, 343-347. doi:10.1016/j. synthmet.2015.08.003

Li, B., Gan, L., Cai, X., Li, X.-L., Wang, Z., Gao, K., et al. (2018). An Effective Strategy toward High-Efficiency Fluorescent OLEDs by Radiative Coupling of Spatially Separated Electron-Hole Pairs. Adv. Mater. Inter. 5 (10), 1800025. doi:10.1002/admi.201800025

\section{AUTHOR CONTRIBUTIONS}

All authors listed have made a substantial, direct, and intellectual contribution to the work and approved it for publication.

\section{FUNDING}

This work is financially supported by the National Key Research and Development Program of China (No. 2016YFB0401303).

Li, J., and Marks, T. J. (2008). Air-stable, Cross-Linkable, Hole-Injecting/ transporting Interlayers for Improved Charge Injection in Organic LightEmitting Diodes. Chem. Mater. 20 (15), 4873-4882. doi:10.1021/cm703689j

Liao, L. S., Klubek, K. P., and Tang, C. W. (2004). High-efficiency Tandem Organic Light-Emitting Diodes. Appl. Phys. Lett. 84 (2), 167-169. doi:10.1063/1.1638624

Lim, S. H., Ryu, G. Y., Seo, J. H., Park, J. H., Youn, S. W., Kim, Y. K., et al. (2008). Dependence of Surface Morphology on Molecular Structure and its Influence on the Properties of OLEDs. Ultramicroscopy 108 (10), 1251-1255. doi:10.1016/ j.ultramic.2008.04.093

Ma, Y.-Y., Hua, X.-C., Zhai, T.-S., Li, Y.-H., Lu, X., Duhm, S., et al. (2019). Doped Copper Phthalocyanine via an Aqueous Solution Process for HighPerformance Organic Light-Emitting Diodes. Org. Electronics 68, 236-241. doi:10.1016/j.orgel.2019.02.019

McEwan, J. A., Clulow, A. J., Nelson, A., Wang, R., Burn, P. L., and Gentle, I. R. (2018). Influence of Dopant Concentration and Steric Bulk on Interlayer Diffusion in OLEDs. Adv. Mater. Inter. 5 (1), 1700872. doi:10.1002/admi. 201700872

Michels, J. J., Zhang, K., Wucher, P., Beaujuge, P. M., Pisula, W., and Marszalek, T. (2021). Predictive Modelling of Structure Formation in Semiconductor Films Produced by Meniscus-Guided Coating. Nat. Mater. 20 (1), 68-75. doi:10.1038/ s41563-020-0760-2

Phatak, R., Tsui, T. Y., and Aziz, H. (2012). Dependence of Dark Spot Growth on Cathode/organic Interfacial Adhesion in Organic Light Emitting Devices. J. Appl. Phys. 111 (5), 054512. doi:10.1063/1.3692390

Salsberg, E., and Aziz, H. (2019). Degradation of PEDOT:PSS Hole Injection Layers by Electrons in Organic Light Emitting Devices. Org. Electronics 69, 313-319. doi:10.1016/j.orgel.2019.03.009

Shi, G., Zhang, X., Wan, M., Wang, S., Lian, H., Xu, R., et al. (2019). Highperformance Inverted Organic Light-Emitting Diodes with Extremely Low Efficiency Roll-Off Using Solution-Processed ZnS Quantum Dots as the Electron Injection Layer. RSC Adv. 9 (11), 6042-6047. doi:10.1039/ c8ra10290b

Singh, A., Nehm, F., Müller-Meskamp, L., Hoßbach, C., Albert, M., Schroeder, U., et al. (2014). OLED Compatible Water-Based Nanolaminate Encapsulation Systems Using Ozone Based Starting Layer. Org. Electronics 15 (10), 2587-2592. doi:10.1016/j.orgel.2014.07.024

Song, C., Hu, Z., Luo, Y., Cun, Y., Wang, L., Ying, L., et al. (2018). Organic/ Inorganic Hybrid EIL for All-Solution-Processed OLEDs. Adv. Electron. Mater. 4 (2), 1700380. doi:10.1002/aelm.201700380

Tang, X., Xiao, S., Fu, Q., Chen, Y., and Hu, T. (2019). Incorporation of Two Electron Acceptors to Improve the Electron Mobility and Stability of Perovskite Solar Cells. J. Mater. Chem. C 7 (27), 8344-8349. doi:10.1039/c9tc02457c

Tsai, C.-T., Gottam, S. R., Kao, P.-C., Perng, D.-C., and Chu, S.-Y. (2020). Improvement of OLED Performances by Applying Annealing and Surface Treatment on Electro-Deposited CuSCN Hole Injection Layer. Synth. Met. 269, 116537. doi:10.1016/j.synthmet.2020.116537

Wang, H., Klubek, K. P., and Tang, C. W. (2008). Current Efficiency in Organic Light-Emitting Diodes with a Hole-Injection Layer. Appl. Phys. Lett. 93 (9), 325. doi:10.1063/1.2978349

Wang, M., Zhu, W., Yin, Z., Huang, L., and Li, J. (2020). Synergistic Effects of LiDoped NiO Film Prepared by Low-Temperature Combustion as Hole-Injection Layer for High Performance OLED Devices. Org. Electronics 85, 105823. doi:10. 1016/j.orgel.2020.105823 
Wang, S., Qiao, M., Ye, Z., Dou, D., Chen, M., Peng, Y., and Wong, W. Y. (2018). Efficient Deep-Blue Electrofluorescence with an External Quantum Efficiency beyond 10. Iscience 9, 532-541. doi:10.1016/j.isci.2018.10.026

Wang, S., Wu, S., Ling, Z., Chen, H., Lian, H., Portier, X., et al. (2020). Mechanically and Thermally Stable, Transparent Electrodes with Silver Nanowires Encapsulated by Atomic Layer Deposited Aluminium Oxide for Organic Optoelectronic Devices. Org. Electronics 78, 105593. doi:10.1016/j.orgel.2019. 105593

Wen, S.-W., Lee, M.-T., and Chen, C. H. (2005). Recent Development of Blue Fluorescent OLED Materials and Devices. J. Display Technol. 1 (1), 90-99. doi:10.1109/jdt.2005.852802

Xie, J., Wang, X., Wang, S., Ling, Z., Lian, H., Liu, N., et al. (2019). Solutionprocessed $\mathrm{ZnO} / \mathrm{MoS} 2$ Quantum Dots Electron Extraction Layer for High Performance Inverted Organic Photovoltaics. Org. Electronics 75, 105381. doi:10.1016/j.orgel.2019.105381

Yadav, R. A. K., Dubey, D. K., Chen, S. Z., Liang, T. W., and Jou, J. H. (2020). Role of Molecular Orbital Energy Levels in Oled Performance. Scientific Rep. 10 (1), 1-15. doi:10.1038/s41598-020-66946-2

Yang, D. Y., Lee, S.-M., Jang, W. J., and Choi, K. C. (2014). Flexible Organic LightEmitting Diodes with $\mathrm{ZnS} / \mathrm{Ag} / \mathrm{ZnO} / \mathrm{Ag} / \mathrm{WO} 3$ Multilayer Electrode as a Transparent Anode. Org. Electronics 15 (10), 2468-2475. doi:10.1016/j.orgel. 2014.06.021

Yu, S.-Y., Chang, J.-H., Wang, P.-S., Wu, C.-I., and Tao, Y.-T. (2014). Effect of ITO Surface Modification on the OLED Device Lifetime. Langmuir 30 (25), 7369-7376. doi:10.1021/la4049659
Zhao, B., Miao, Y., Wang, Z., Wang, K., Wang, H., Hao, Y., et al. (2016). High Efficiency and Low Roll-Off Green OLEDs with Simple Structure by Utilizing Thermally Activated Delayed Fluorescence Material as the Universal Host. Nanophotonics 6 (5), 1133-1140. doi:10.1515/nanoph2016-0177

Zhao, X., Chen, J., and Park, N. G. (2019). Importance of Oxygen Partial Pressure in Annealing $\mathrm{NiO}$ Film for High Efficiency Inverted Perovskite Solar Cells. Sol. RRL 3 (4), 1800339. doi:10.1002/solr. 201800339

Zhao, Y., Wu, S., Ling, Z., Chen, H., Yu, N., Zhou, P., et al. (2020). Systematical Investigation of Ultrathin Doped Emissive Layer Structure: Achieving Highly Efficient and Long-Lifetime Orange Organic Light-Emitting Diodes. Adv. Mater. Inter. 7 (2), 1901609. doi:10.1002/admi.201901609

Conflict of Interest: The authors declare that the research was conducted in the absence of any commercial or financial relationships that could be construed as a potential conflict of interest.

Copyright (C) 2021 Zhu, Ding, Yi, Chen, Wei, Huang and Li. This is an open-access article distributed under the terms of the Creative Commons Attribution License (CC $B Y)$. The use, distribution or reproduction in other forums is permitted, provided the original author(s) and the copyright owner(s) are credited and that the original publication in this journal is cited, in accordance with accepted academic practice. No use, distribution or reproduction is permitted which does not comply with these terms. 